\title{
Urachal Cyst
}

National Cancer Institute

\section{Source}

National Cancer Institute. Urachal Cyst. NCI Thesaurus. Code C85216.

A cyst that develops from the remnants of the urachus. Since urachal cysts are noncommunicating, they are usually asymptomatic unless infected. 